DOI: http://dx.doi.org/10.5007/2175-7941.2014v31n1p124

\title{
Projeto de um sensor de pressão manométrica para ensino de física em tempo real ${ }^{+*}$
}

Fábio Saraiva da Rocha

Centro de desenvolvimento Tecnológico

Universidade Federal de Pelotas

Pelotas - RS

Paulo Henrique Guadagnini

Universidade Federal do Pampa

Bagé - RS

\section{Resumo}

Apresentamos o projeto de um sensor de pressão manométrica voltado ao ensino de física experimental. Este dispositivo permite a medida da pressão de um sistema gasoso em comparação com a pressão atmosférica local. O sensor é composto de um transdutor de pressão piezoresistivo e uma placa de desenvolvimento Arduíno para digitalização de leituras analógicas do transdutor, tratamento dos dados e comunicação digital através da interface USB. O sensor de pressão permite que os dados de pressão possam ser transferidos a um computador e acompanhados ao longo da execução de um experimento, com a apresentação de tabelas e gráficos em tempo real (Física em Tempo Real - FTR), utilizando a ferramenta de aquisição de dados PLX-DAQ e a planilha eletrônica Excel. Apresentamos, inicialmente, as características físicas e operacionais do transdutor MPX4250GP, aspectos gerais da placa Arduíno e detalhamos a programação computacional envolvida em todo o projeto. Discutimos o desempenho e limitações deste sensor

\footnotetext{
Design of a gauge pressure sensor for Physics teaching in real time

* Recebido: agosto de 2013.

Aceito: novembro de 2013.
} 
quando comparado a um sensor comercial GPS-BTA da marca Vernier. Apresentamos a possibilidade de uso deste dispositivo em uma prática ilustrativa com balões de látex dispostos num sistema fisico que pode ser facilmente reproduzido em um laboratório didático de física experimental ou mesmo numa sala de aula comum. O sensor de pressão apresentou desempenho similar ao sensor comercial GPS-BTA, e seu uso se mostra promissor em práticas de ensino de física que busquem a inovação no laboratório didático, a possibilidade de utilizar o recurso da FTR com uma classe demonstrativa, ou pelo envolvimento de estudantes em projetos interdisciplinares.

Palavras-chave: Ensino de Física. Sensor de Pressão. Instrumentação. Aquisição automática de dados.

\begin{abstract}
We present the design of a gange pressure sensor aimed at teaching experimental Physics. This device enables reading the pressure of a gaseous system in comparison to local atmospheric pressure. The sensor consists of a piezoresistive pressure transducer and Arduino development board for digitizing analog readings from the transducer, data processing and digital communication via the USB interface. The pressure sensor provides real time numerical data (Real Time Physics - RTP) for measuring pressure through tables and graphs using the PLX-DAQ data acquisition tool and the Excel spreadsheet. We show the physical and operational characteristics of the MPX4250GP transducer, the general aspects of the Arduino board and we detail the computer programming involved in the entire project. We discuss the performance and limitations of this sensor when compared to a commercial sensor GPS-BTA Vernier brand. The reader can use this device in a practice with illustrative latex balloons arranged in a physical system that can be easily reproduced in an experimental Physics laboratory or even in an ordinary classroom situation. The pressure sensor performance was similar to the commercial sensor GPS-BTA, and it shows promise for the use in Physics teaching practices that seek
\end{abstract}


innovation in the teaching laboratory, the possibility of using the $R T P$ resource with class demonstrations, or the involvement of students in interdisciplinary projects.

Keywords: Physics Teaching. Pressure Sensor. Probeware. Real Time Physics.

\section{Introdução}

Atualmente temos visto o surgimento de diversos equipamentos comerciais (sistemas proprietários) projetados para ensino de física experimental, que podem custar milhares de dólares se levarmos em conta custos de importação, transporte, impostos e demais taxas. Verdadeiras "caixas pretas" especialmente construídas para uso nos laboratórios didáticos de ensino de física (<www.vernier.com>, <www.pasco.com>, <www.cidepe.com.br $>$ ). Tais equipamentos contam com ótima tecnologia e desempenho, e são projetados para uso a partir de conexões diretas com computadores, principalmente pela porta serial, pela porta USB (Universal Serial Bus) e através de conexão sem fio. Estes instrumentos didáticos são projetados, na maioria dos casos, a partir de componentes eletrônicos e módulos sensores de baixo custo financeiro, baseados em princípios físicos bem conhecidos.

O mercado internacional tem disponibilizado módulos sensores que, se interligados a uma eletrônica adequada, podem medir diversas grandezas físicas. Estes módulos eletrônicos sensores podem ser comandados por dispositivos chamados microcontroladores (também popularizados e vendidos hoje a baixo custo) que executam tarefas de processamento de dados matemáticos, conversão entre sinais analógicos e digitais, envio e recebimento de comandos de um computador através uma conexão física devidamente projetada.

Embora já exista uma comunidade de pesquisadores voltada ao desenvolvimento de projetos de instrumentação eletrônica de baixo custo, baseados em módulos sensores, para uso em laboratórios didáticos de ensino de ciências (GUADAGNINI; BARLETTE, 2005; ROCHA; GUADAGNINI, 2009; GUADAGNINI; ROCHA et al., 2011), o número de projetos sobre esses sistemas automáticos de aquisição e tratamento de dados assistidos por computador ainda é pequeno para estimular professores e alunos do ensino superior ou mesmo ensino médio a reproduzirem tais projetos e construírem seus próprios equipamentos.

O projeto apresentado aqui tem por finalidade mostrar a construção de um protótipo de sensor de pressão baseado em um módulo transdutor de pressão inte- 
grado e um microcontrolador. São apresentadas as características físicas do transdutor de pressão, a constituição básica do projeto, desde componentes eletrônicos até a programação computacional envolvida em todo o processo. Apontamos uma possibilidade de uso em uma prática demonstrativa utilizando balões de látex (LANG; LEVIN, 2004). O dispositivo transdutor transmite informações por sua saída de tensão elétrica variável que pode ser digitalizada através de uma placa Arduíno que tem um microcontrolador embarcado.

Projetos como o que apresentamos neste trabalho tem a intenção de promover maior acessibilidade às tecnologias disponíveis atualmente e suprir parte da demanda de renovação do instrumental de coleta de dados experimentais para professores e escolas que não podem contar com grandes recursos financeiros para a compra de equipamentos comerciais modernos.

\section{Ensino de Física em Tempo Real (FTR)}

O protótipo desenvolvido como produto deste projeto tem conexão direta com o computador, sendo possível a apresentação dos dados coletados de uma experiência em tempo real (Física em Tempo Real - FTR) (SOKOLOFF; LAWS et al., 2007). Através desta importante ferramenta de laboratório obtemos excelentes possibilidades de abordagens didáticas que, aliadas ao trabalho docente e guias reflexivos de laboratório, tendem a levar o estudante a sentir-se estimulado e envolvido ativamente na sua própria aprendizagem (METCALF; TINKER, 2004). As experiências de outros pesquisadores (BORGES, 2002; HAAG; ARAUJO et al., 2005) sugerem vários benefícios de se utilizar aquisição automática de dados no ensino de ciências, tais como o enriquecimento das experiências de aprendizagem, a possibilidade de se efetuar experimentos em que a coleta de dados é efetuada em intervalos de tempo muito pequenos (frações de segundo) ou muito longos (horas), agilização da coleta dos dados com mais tempo para sua análise e interpretação, e a alfabetização científica e tecnológica que decorre da experiência prática de se utilizar sistemas de aquisição automática de dados.

A utilização de sensores conjuntamente com sistemas de aquisição automática de dados em tempo real na construção de práticas de laboratório de física tem representado inovação nas formas de ensinar e de aprender ainda muito pouco difundidas, e contribui na difusão de novas práticas integrando mais tecnologia, teoria e experimento. Os objetivos buscados através de projetos como o que é apresentado neste trabalho serão fortemente incentivados quando programas governamentais como "Um Computador por Aluno" (UCA) $(<$ http://www.uca.gov.br/institucional/projeto.jsp $>)$ torne amplamente acessível o 
uso de tecnologias computacionais no ambiente educacional por alunos das escolas públicas brasileiras, induzindo a mudanças nas práticas de alunos e seus professores.

As atividades de FTR são adequadas para compor um ambiente de aprendizagem ativa, e podemos resumir, conforme Sokoloff e Benegas (SOKOLOFF; BENEGAS, 2009), as principais diferenças entre a aprendizagem passiva e aprendizagem ativa (FTR) através da Tabela 1.

Tabela 1. Características da Aprendizagem Passiva em oposição a Aprendizagem Ativa.

\begin{tabular}{|c|c|}
\hline Aprendizagem Passiva & Aprendizagem Ativa \\
\hline $\begin{array}{l}\text { Professor e livro são as autoridades e } \\
\text { fontes de conhecimento. }\end{array}$ & $\begin{array}{l}\text { Os estudantes constroem seu conheci- } \\
\text { mento desenvolvendo atividades. A } \\
\text { observação do mundo real é a autoridade } \\
\text { e a fonte do conhecimento. }\end{array}$ \\
\hline $\begin{array}{l}\text { As crenças dos alunos não são explici- } \\
\text { tamente desafiadas. }\end{array}$ & $\begin{array}{l}\text { Utiliza um ciclo de aprendizagem que } \\
\text { desafia os estudantes a comparar suas } \\
\text { previsões. (baseadas em suas crenças) } \\
\text { com o resultado dos experimentos. }\end{array}$ \\
\hline $\begin{array}{l}\text { Os estudantes não se dão conta das } \\
\text { diferenças entre suas crenças e o que é } \\
\text { dito pelo professor. }\end{array}$ & $\begin{array}{l}\text { Os estudantes modificam suas crenças } \\
\text { quando veem as diferenças entre elas e } \\
\text { suas próprias observações. }\end{array}$ \\
\hline O papel do professor é de autoridade. & $\begin{array}{l}\text { O professor é um guia do processo de } \\
\text { aprendizagem. }\end{array}$ \\
\hline $\begin{array}{l}\text { Não estimula a colaboração dos alu- } \\
\text { nos. }\end{array}$ & $\begin{array}{l}\text { Estimula a colaboração entre os estu- } \\
\text { dantes. }\end{array}$ \\
\hline $\begin{array}{l}\text { Nas aulas se apresentam os "feitos" da } \\
\text { física sem referência a experimentos. }\end{array}$ & $\begin{array}{l}\text { Os resultados dos experimentos reais } \\
\text { são observados de forma compreensível. }\end{array}$ \\
\hline $\begin{array}{l}\text { O laboratório é utilizado para confir- } \\
\text { mar o "aprendido". }\end{array}$ & $\begin{array}{l}\text { O laboratório é utilizado para aprender } \\
\text { conceitos. }\end{array}$ \\
\hline
\end{tabular}

As atividades com FTR geralmente envolvem experimentos conceituais, e são projetadas de modo que os alunos sejam conduzidos ao despertar dos conceitos físicos básicos a partir de suas observações experimentais e interação e colaboração entre eles. A dinâmica das atividades de FTR inclui um ciclo de aprendizagem utilizado no estudo de cada conceito físico, em que os alunos: 
- são introduzidos ao tema de estudo e ao projeto do experimento;

- são questionados sobre as suas previsões com respeito aos resultados que devem ser obtidos no experimento;

- são incentivados a interagirem com seus pares (pequenos grupos) no sentido de discutir as previsões sobre os resultados experimentais;

- efetuam o experimento e observam os resultados, em geral representados na forma de tabelas e gráficos gerados em tempo real utilizando sensores e sistemas de aquisição automática de dados;

- confrontam os resultados obtidos com as previsões feitas anteriormente, e tentam explicar os fenômenos observados.

- são desafiados a prever o comportamento de sistemas físicos diferentes dos abordados nos experimentos, mas que são baseados nos mesmos conceitos básicos estudados.

Sokoloff e Benegas (SOKOLOFF; BENEGAS, 2009) e Sokoloff (SOKOLOFF, 2012) apresentam possibilidades de uso da FTR utilizando atividades demonstrativas, onde apenas um computador (do professor) é necessário, atividades em grupos de três ou quatro alunos e atividades para serem desenvolvidas individualmente, por cada aluno. O ciclo de aprendizagem acima também pode ser adaptado para compor aulas demonstrativas interativas, nas quais o experimento é efetuado pelo professor perante o grande grupo de alunos, tornando possível configurar um ambiente de aprendizagem ativa nas salas de aulas.

Do ponto de vista educacional, o simples uso de recursos tecnológicos não garante vantagens no aprendizado, mas podem potencializar a aprendizagem quando utilizados em conjunto com estratégias instrucionais adequadas. As possibilidades pedagógicas dessas abordagens vão mais além, pois oportunizam formas de explorar os fenômenos naturais em situações concretas de aprendizagem de maneira integrada à teoria, na perspectiva de integração teoria-experimento apontada por Gil Pérez et al. (PÉREZ; MÁS et al., 1999), rompendo com a histórica separação entre a teoria e o experimento nos currículos escolares. A observação e a análise de um fenômeno em tempo real que essas tecnologias propiciam têm o benefício de favorecer o confronto de concepções e ideias, o desenvolvimento e teste de novos conhecimentos científicos no momento e no ambiente em que os fenômenos são produzidos, promovendo a reflexão e o questionamento, competências essenciais para a promoção da autonomia.

A abordagem da FTR permite ampliar a função tradicional do laboratório didático dos currículos atuais, caracterizada como complementar às aulas teóricas ou com ênfase na aprendizagem procedimental, tornando o laboratório um espaço 
de aprendizagem mais dinâmico, participativo e integrado as demais disciplinas, em especial propiciando um vínculo entre teoria e experimento.

\section{Aspectos físicos de um transdutor de pressão piezoresistivo}

A pressão é uma grandeza física escalar definida como a razão entre a força e a área sobre a qual a referida força é aplicada perpendicularmente. Considerando que a força $F$ é distribuída uniformemente sobre a área $A$, a pressão $P$ pode ser expressa segundo a equação:

$$
P=\frac{F}{A}
$$

A pressão é medida na unidade do SI chamada pascal $(\mathrm{Pa})$ e corresponde a $1 \mathrm{~N} / \mathrm{m}^{2}$. A pressão manométrica $P$ pode ser definida como a pressão relativa à pressão atmosférica local $P_{\text {atm }}$ segundo:

$$
P=P_{a b s}-P_{a t m}
$$

em que $P_{a b s}$ é a pressão absoluta, referenciada a um vácuo perfeito.

Neste trabalho, denotaremos de sensor o sistema de medida composto de transdutor, circuito de condicionamento de sinal, conversor analógico-digital e interface de comunicação para transmissão dos dados digitalizados para um computador. Um elemento importante de um sensor é o transdutor, que tem como função converter uma variável de medida física, como a pressão manométrica, em uma variável de sinal analógica, como a tensão elétrica. Na saída do transdutor temos uma tensão elétrica analógica, continuamente variável, que pode ser convenientemente processada por circuitos eletrônicos que efetuam o condicionamento do sinal e conversão para o domínio digital através de um conversor analógicodigital.

O transdutor de pressão utilizado no projeto do sensor de pressão manométrica descrito neste trabalho é um dispositivo fabricado utilizando a tecnologia MEMS (Micro-Electro-Mechanical Systems ou sistemas microeletromecânicos) que possibilita a montagem de estruturas mecânicas e eletrônicas através de técnicas similares às utilizadas na produção de circuitos integrados (SZE, 1994). O transdutor de pressão possui um microcircuito que inclui elementos piezoresistivos sensíveis a deformações, resistores, termistores e um sistema de amplificação de sinais. O microcircuito interno tem as funções de excitação dos elementos ativos (piezoresistores), compensação da saída de sinal devido a variações de temperatura ambiente e amplificação do sinal elétrico analógico de modo que sua saída possa 
ser facilmente conectada à entrada de um conversor analógico-digital. Uma representação esquemática de um corte transversal da estrutura física do transdutor de pressão é apresentada na Fig. 1 .

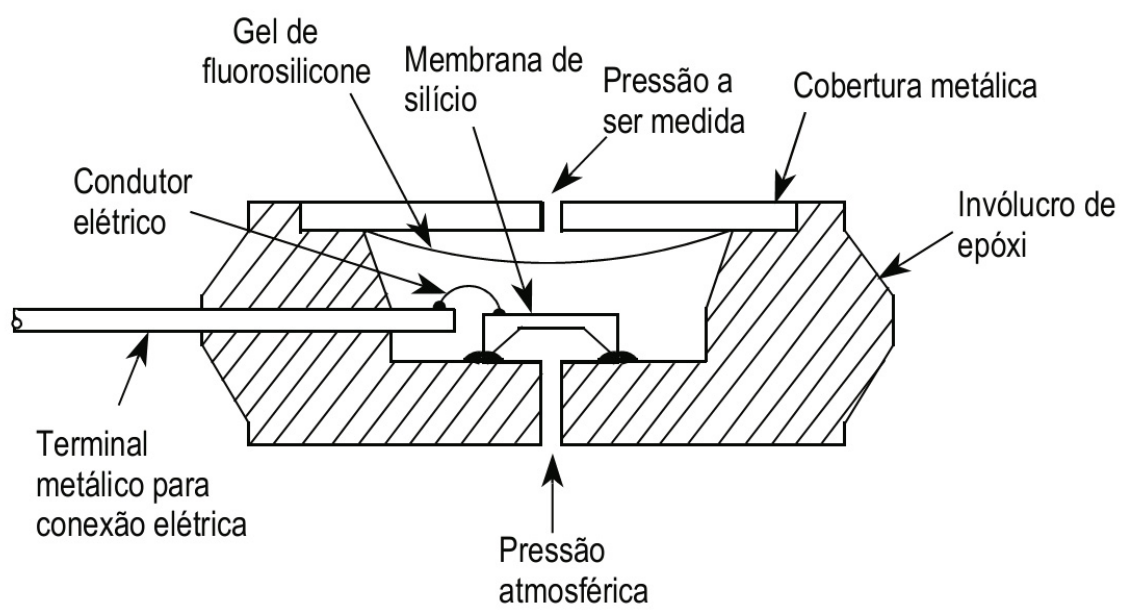

Fig. 1 - Corte transversal mostrando a estrutura interna do transdutor de pressão piezoresistivo MPX4250GP. Figura adaptada do manual do fabricante.

Os elementos ativos do transdutor são piezoresistores compostos de uma fina membrana de silício microcristalino semicondutor que sofre deflexão quando sujeito à pressão externa. A deformação da rede cristalina do silício causa alterações na mobilidade e número de portadores de carga no cristal, alterando a sua resistividade elétrica (piezoresistividade). Um provável mecanismo que contribui para este efeito é a alteração da diferença de energia entre as bandas de valência e condução do silício devido a variações de volume da amostra (SZE, 1994). O piezoresistor comporta-se como um resistor cuja resistência varia com a sua deformação mecânica, permitindo que o transdutor de pressão converta a energia mecânica, aplicada em sua membrana, em um sinal elétrico caracterizado por uma diferença de potencial elétrico.

O transdutor é suportado por um invólucro de epóxi coberto por uma capa metálica contendo um orifício para entrada do gás que transmite a pressão a ser medida. Uma camada flexível de fluorosilicone protege a membrana de silício contendo o microcircuito do transdutor, permitindo a transmissão da pressão a ser medida até a membrana de silício. A pressão atmosférica é aplicada através do 
orifício na parte inferior do invólucro de modo que esta atue na parte inferior da membrana, fazendo com que a membrana sofra deformações líquidas resultantes da diferença das pressões $P_{a b s}$ e $P_{a t m}$, conforme a equação (2).

$\mathrm{O}$ transdutor de pressão piezoresistivo é produzido industrialmente utilizando tecnologias de microfabricação em superfície, em que a membrana de silício do transdutor de pressão é suportada por um aro de silício grosso e integra na sua estrutura, através de processos de difusão e litografia, os piezoresistores e outros componentes eletrônicos que constituem o transdutor. Os piezoresistores são usualmente posicionados nos locais da membrana sujeitos a maior deformação, como próximo das bordas. A Fig. 2 mostra esquematicamente uma membrana de silício de um transdutor de pressão contendo um piezoresistor sofrendo deformação devido à aplicação de uma pressão externa. Quando uma diferença de pressão é aplicada através da membrana, ele irá dobrar para baixo ou para cima, causando tração ou compressão nos piezoresistores. A variação de resistência causada por esta deformação é medida utilizando um circuito elétrico do tipo ponte de Wheatstone, em que um ou mais piezoresistores são ligados nos braços da ponte.

a)

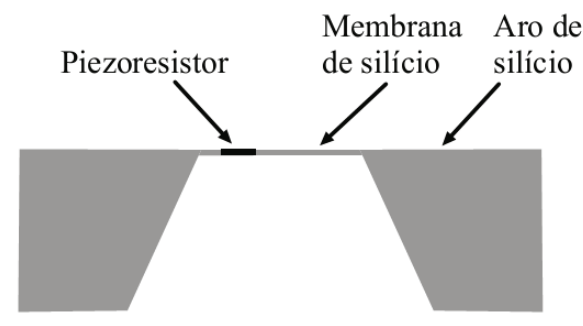

b)

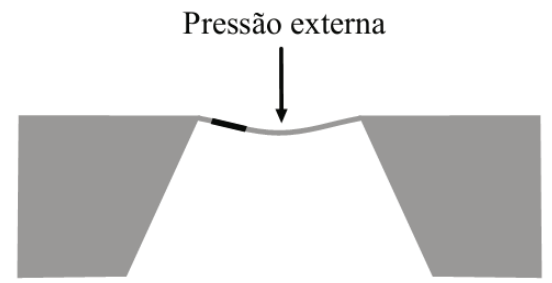

Fig. 2 -Representação esquemática da membrana de silício de um transdutor de pressão piezoresistivo sofrendo deformação devido à aplicação de uma pressão externa. 
Durante a deformação da membrana de silício, cada piezoresistor é também deformado, o que leva ao aparecimento de tensões mecânicas longitudinais e transversais no mesmo, definidas como a intensidade da força interna agindo no corpo do piezoresistor por unidade de área de sua seção transversal. Assumindo que a tensão mecânica exercida sobre a membrana de silício é constante através dos piezoresistores, a variação relativa de resistência elétrica $\Delta R$ devido à tensão longitudinal $\sigma_{l}$ e transversal $\sigma_{t}$ é:

$$
\frac{\Delta R}{R}=\sigma_{\mathrm{t}} \pi_{\mathrm{t}}+\sigma_{\mathrm{t}} \pi_{\mathrm{t}}
$$

em que $\pi_{1}$ e $\pi_{t}$ são os coeficientes piezoresistivos longitudinal e transversal, que são característicos dos piezoresistores. O transdutor de pressão é usualmente construído posicionando-se quatro piezoresistores sobre o campo de tensões da membrana de silício de modo a possibilitar a aplicação de uma conexão do tipo ponte de Wheatstone, conforme a Fig. 3 (KIRIANAKI; YURISH et al., 2002).

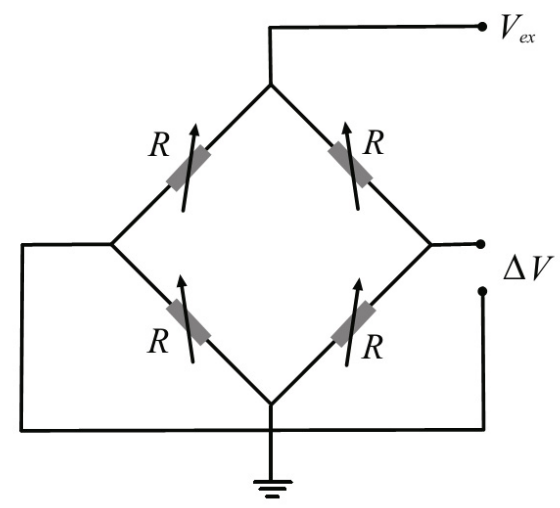

Fig. 3 - Representação esquemática de parte do circuito elétrico interno do transdutor de pressão, mostrando uma ponte de Wheatstone contendo quatro piezoresistores.

A configuração da ponte de Wheatstone possui a vantagem de converter a mudança na resistência $\Delta R$ diretamente em um sinal de tensão elétrica $\Delta V$ segundo:

$$
\Delta V=\frac{\Delta R}{R} V_{e x}
$$

em que $R$ é a resistência de cada piezoresistor na ausência de tensão, e $V_{\text {ex }}$ é a tensão de excitação da ponte. Segundo as equações (3) e (4), é esperado que o sinal 
de saída do transdutor apresente uma dependência linear das tensões atuando sobre a membrana de silício:

$$
\Delta V=\left(\sigma_{\imath} \pi_{\mathrm{t}}+\sigma_{\mathrm{t}} \pi_{\mathrm{t}}\right) V_{e x}
$$

O transdutor de pressão piezoresistivo é projetado de modo que variações na tensão mecânica aplicada sobre o diafragma de silício produzam um sinal de saída proporcional à tensão mecânica aplicada. A pressão diferencial que o transdutor é submetido (equação 2) é relacionada linearmente com a tensão elétrica de saída segundo uma função de transferência do tipo:

$$
P=\frac{\Delta V}{s}+b
$$

em que $s$ é definido como a sensibilidade do transdutor, sendo uma medida da variação da tensão elétrica de saída por unidade de pressão aplicada, e $b$ é uma correção para um erro sistemático devido à presença de uma leitura residual quando o transdutor é submetido a uma pressão manométrica nula.

Deve-se enfatizar que, em um transdutor piezoresistivo, a sua resposta é devida, essencialmente, à alteração de sua resistividade com a deformação, diferentemente de um transdutor do tipo piezoelétrico que responde a deformações mecânicas produzindo, sem a necessidade de excitação elétrica, uma diferença de potencial que pode ser amplificada.

\section{Projeto do sensor eletrônico de pressão manométrica}

Os módulos transdutores de pressão utilizados na prática demonstrativa que apontaremos neste trabalho foram obtidos gratuitamente pelo endereço eletrônico da empresa Freescale ( $w w w$.freescale.com). Esta empresa disponibiliza até cinco amostras do dispositivo e envia pelo custo de cinco dólares americanos depois de aprovado o mérito da solicitação. Veja a foto do sensor na Fig. 4.

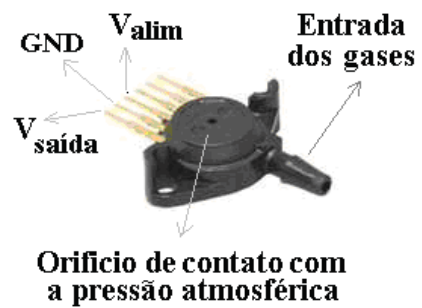

Fig. 4 - Módulo sensor manométrico MPX4250GP fabricado pela empresa Freescale. 
Na Fig. 4 é possível notar os três pinos de soldagens para os fios de alimentação GND (ground) de potencial elétrico baixo e $V_{\text {alim }}$ que deve receber a tensão elétrica contínua e estabilizada dentro da banda de valores que pode ir de $4,85 \mathrm{~V}$ até $5,35 \mathrm{~V}$. O valor típico de alimentação indicado pelo fabricante é de 5,1 $\mathrm{V}$ e a corrente elétrica típica prevista é de 7,0 mA. Quando devidamente alimentado, o pino $V_{\text {saida }}$ deve apresentar uma tensão elétrica CC (corrente contínua) variável (dependente da pressão) de até $5,0 \mathrm{~V}$ que representará o fundo de escala de $250 \mathrm{kPa}$. Os demais três pinos que aparecem na Fig. 4 não tem nenhum contato elétrico previsto pelo fabricante.

A Fig. 5 apresenta o esquema elétrico de ligação recomendado pelo fabricante para desacoplamento do circuito de alimentação do módulo sensor e o circuito de interfaceamento com o conversor $\mathrm{A} / \mathrm{D}$ de um microcontrolador. Este último circuito serve para filtragem do sinal elétrico de saída do módulo sensor.

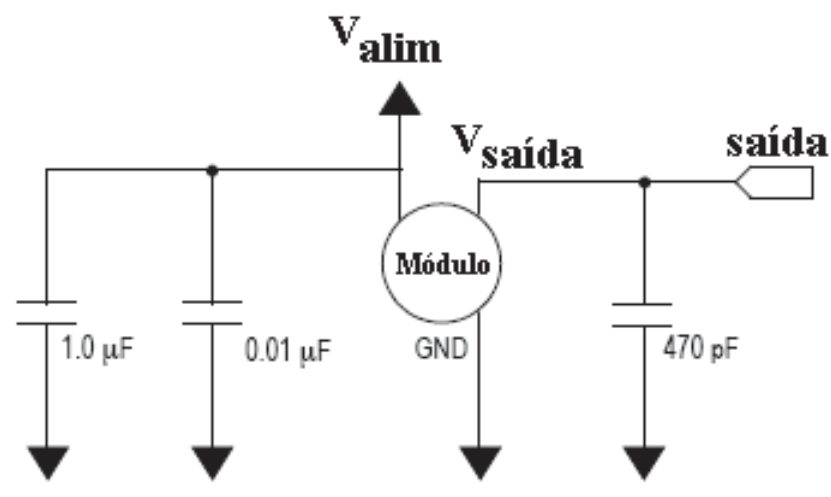

Fig. 5 - Esquema elétrico de alimentação do módulo sensor e filtragem do sinal elétrico de saída. Figura adaptada do manual do fabricante.

Este dispositivo pode atuar dentro de uma faixa de temperatura que vai de $0^{\circ} \mathrm{C}$ até $85^{\circ} \mathrm{C}$ e inclui compensação automática para variações de temperatura que possam ocorrer durante uma medida. O dispositivo sensor é calibrado na origem da fabricação o que garante o desempenho preconizado pelo fabricante dentro dos limites de uso apontados. Veja a curva de calibração na Fig. 6. 


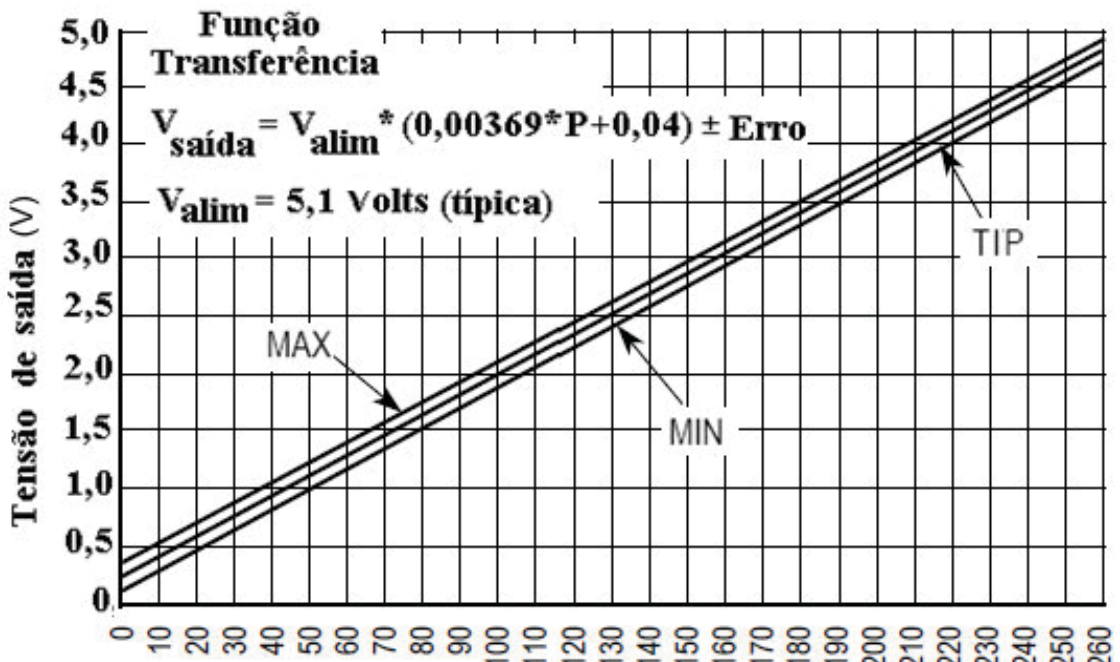

\section{Pressão (kPa)}

Fig. 6 - Tensão elétrica CC de saída do módulo sensor em função da variação de pressão na entrada de gás do dispositivo. Também é mostrada a curva de saída para os casos de tensão elétrica de alimentação mínima (MIN), típica (TIP) e máxima (MAX) previstas para o dispositivo. Figura adaptada do manual do fabricante.

A sensibilidade $(\Delta V / P)$ típica informada pelo fabricante se apresenta com valor de $18,8 \mathrm{mV} / \mathrm{kPa}$. Importante atentar que os valores típicos de saída apresentados na Fig. 6 só serão observados se a alimentação do dispositivo também for a típica informada pelo fabricante. Saindo deste valor de alimentação, a sensibilidade $(\mathrm{mV} / \mathrm{kPa})$ varia crescendo ou decrescendo linearmente. A maior pressão que o módulo suporta de sobrecarga é $1000 \mathrm{kPa}$. No interior da Fig. 6 aparece a função de transferência que foi usada na programação do sensor e que aponta qual a pressão que o módulo estará sujeito quando sua saída de sinal elétrico estiver apresentando os valores do gráfico.

\section{A plataforma Arduíno}

O sinal de tensão elétrica de saída analógica variável $\mathrm{CC}$ do sensor de pressão utilizado neste trabalho pode ser convenientemente lido através de uma placa Arduíno (por exemplo, na versão Nano) equipada com o microcontrolador 
ATmega 328. O microcontrolador é constituído de um microprocessador, memória, conversor analógico-digital e portas de entrada e saída do dispositivo.

O Arduíno é uma plataforma de programação não proprietária, muito útil para o desenvolvimento de projetos envolvendo módulos sensores e/ou de uso geral em práticas voltadas ao ensino de física experimental (CAVALCANTE; TAVOLARO et al., 2011; SOUZA; PAIXÃO et al., 2011). A versão Nano pode ser acoplada ao sensor de pressão através de um cabo elétrico de apenas três fios dedicados para a alimentação do sensor e leitura do sinal elétrico de saída. Por sua vez, a placa Arduíno se liga fisicamente com um computador através do cabo miniB USB e a porta serial. O cabo mini-B USB além de permitir a transferência de dados proporciona a alimentação da placa Arduíno. A placa Arduíno também fornece alimentação 5,0 V para a alimentação do módulo sensor.

Como se pode ver na Fig. 7, a versão Nano tem oito entradas analógicas, cada uma com 10 bits de resolução que permitem uma amplitude de 1024 valores diferentes numa conversão analógico-digital. Por padrão estas portas medem de $0 \mathrm{a}$ $5,0 \mathrm{~V}$, embora seja possível mudar o limite superior usando o pino de referência analógica (Ref. Analógica). São 14 pinos digitais que podem operar como entrada ou saída conforme a programação desejada. Alguns desses pinos, que representam portas digitais, podem ser reconfigurados para atuarem como saídas analógicas, através de modulação por largura de pulso, ou Pulse-Width Modulation (PWM). Veja na Fig. 7 uma visão geral da placa Arduíno.

O microcontrolador embarcado na placa Arduíno, ATmega328, pode ser programado através de um software específico fornecido sem custos pela comunidade desenvolvedora da plataforma. A linguagem de programação disponível para o usuário é baseada nas linguagens $\mathrm{C} / \mathrm{C}++$ (ver $<$ http://Arduíno.cc/en/Main/Software $>$ ). O envio de códigos para o microcontrolador sem o uso de um gravador de hardware externo é uma facilidade encontrada nesta placa. Certamente o leitor poderá verificar na literatura inúmeros exemplos de aplicações envolvendo a placa Arduíno, que vão desde robótica e automação industrial até ensino de física (SOUZA; PAIXÃO et al., 2011).

O anexo apresenta o código escrito para leitura do sensor de pressão e a programação utilizada para envio de informações via porta serial e disponibilização dos dados pelo programa Excel através de gráficos em tempo real. 


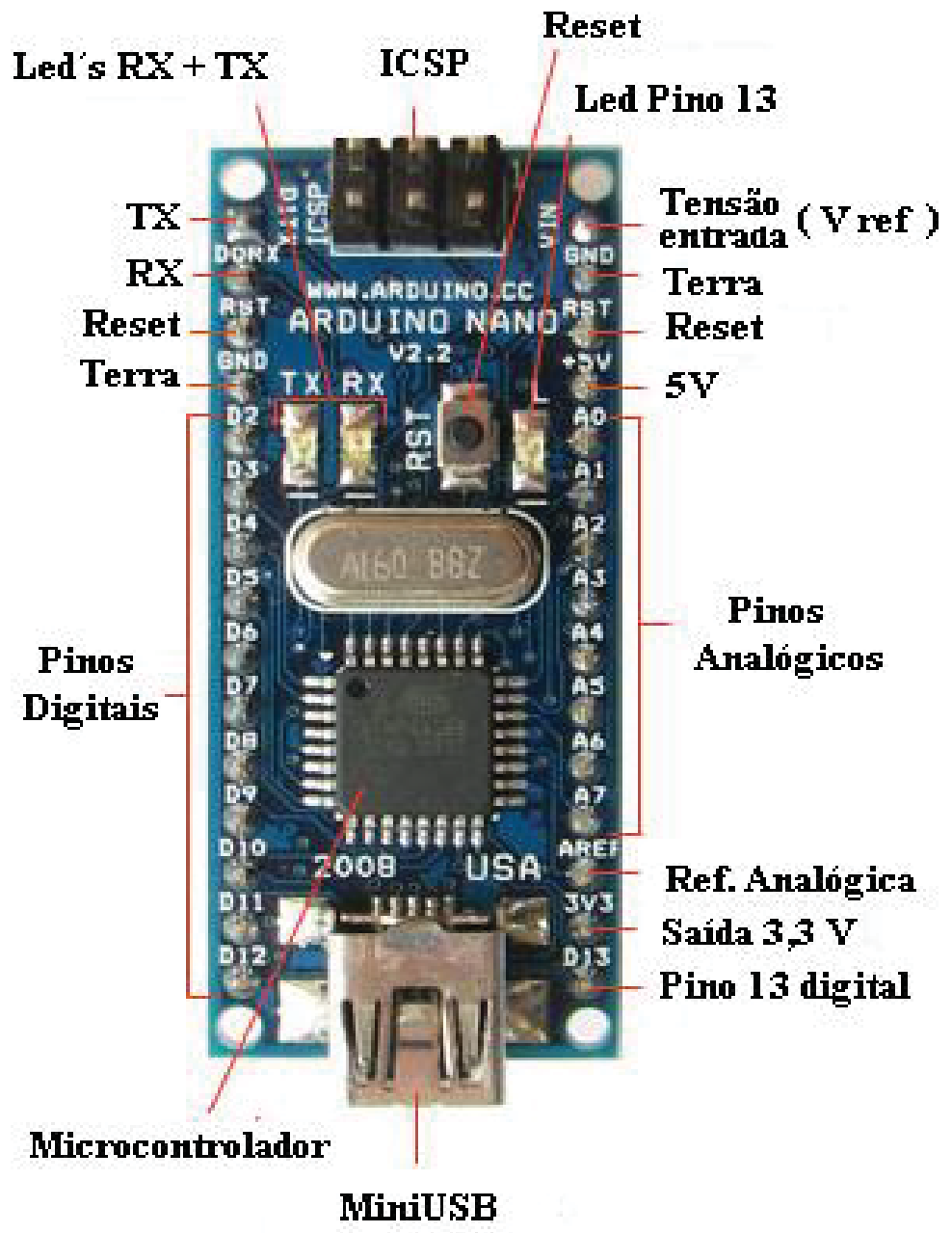

Fig. 7 - Visão geral da placa Arduíno Nano. Adaptado do endereço $<$ http://Arduíno.cc/en/Main/ArduínoBoardNano>.

Na Fig. 7, ICSP (In-Circuit Serial Programming) é a entrada para reprogramação do microcontrolador e as siglas (RX) e (TX) apontam os pinos de recebimento e transmissão de dados na forma serial respectivamente. Veja mais detalhes em <http://Arduíno.cc/en/Main/ArduínoBoardNano $>$. 


\section{Sistema de medida e teste de desempenho}

Apresentamos a seguir uma aplicação de uso do sensor para monitorar as variações de pressão ocorridas no interior de um circuito fechado de gases que conecta dois balões de látex (reservatórios), um no formato esférico, modelo globo, marca Regina e outro modelo espagueti da marca Chocomar. O circuito fechado possui duas válvulas de três vias para regular a entrada e saída de ar dos balões e uma válvula central de duas vias que serve apenas para interromper a via de comunicação de gás entre os reservatórios. Estas válvulas de três vias permitem que os balões sejam inflados independentemente com ar até estabelecermos as pressões internas que desejarmos. Os balões de látex foram fixados em seringas plásticas simples de $10 \mathrm{~mL}$ que foram adaptadas para tal finalidade, conforme mostra a Fig. 8. Estas seringas por sua vez foram adequadamente conectadas nas válvulas de três vias e estas interconectadas por tubos plásticos utilizados em equipos de soro. A Fig. 8 apresenta os itens principais do circuito montado especificamente para testar o sensor apresentado neste trabalho. Em (a) a válvula de duas vias, em (b) de três vias, em (c) a seringa plástica de $10 \mathrm{~mL}$ cortada em sua base para fixação dos balões e em (d) o equipo de soro de onde foram retirados os tubos plásticos que interligam todo o sistema de gás. Todo este material pode ser adquirido por baixo custo em empresas de distribuição de equipamentos hospitalares ou mesmo em simples farmácias.

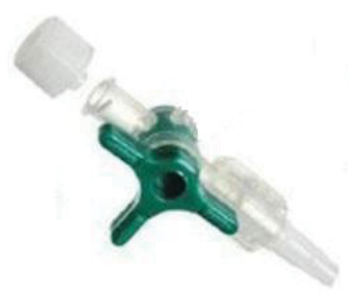

(a)

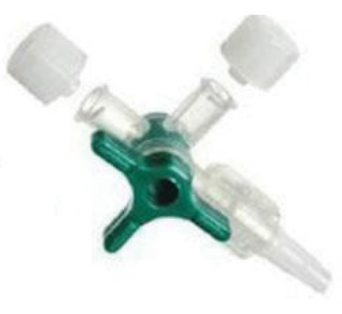

(b)

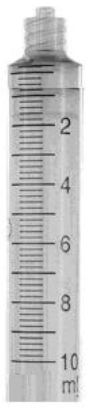

(c)

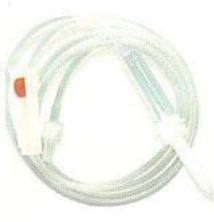

(d)

Fig. 8 - Itens para montagem do circuito fechado que conecta os balões de látex. Em (a) a válvula de duas vias, em (b) de três vias, em (c) a seringa plástica de $10 \mathrm{~mL}$ cortada em sua base para fixação dos balões e em (d) o equipo de soro de onde foram retirados os tubos plásticos que interligam todo o sistema. 
A Fig. 9 mostra uma foto do sistema montado com os balões e os sensores interligados através das válvulas regulatórias.

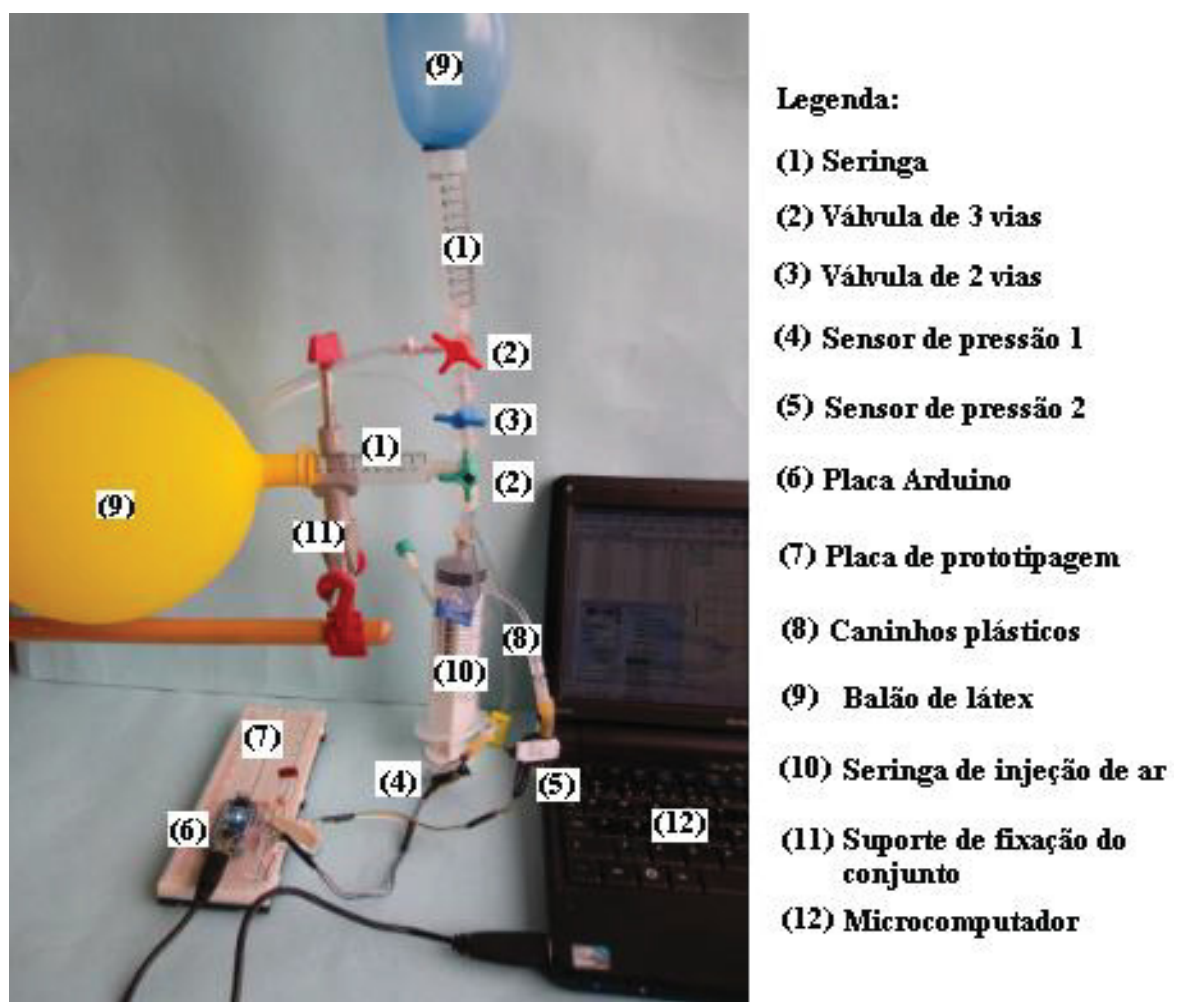

Fig. 9 - Equipamento montado.

Como teste de desempenho para os sensores de pressão deste trabalho, propomos reproduzir parcialmente a experiência indicada por (LANG; LEVIN, 2004; LEVIN; LANG, 2004) utilizando para isso também um sensor comercial modelo GPS-BTA da marca Vernier como referência de desempenho. No trabalho anteriormente citado, dois balões de látex, ambos com o mesmo tamanho original e desigualmente inflados, são conectados às extremidades de uma mangueira, na qual uma válvula de obstrução impede a passagem de ar. Os autores demonstram que, depois de encherem os balões com volumes diferentes de ar, quando a obstrução é removida, o balão menor tanto poderá inflar quanto desinflar ou até mesmo permanecer com o volume inalterado. Os autores concluem que a possibilidade que 
se concretizará depende dos volumes e pressões iniciais dos dois balões.

$\mathrm{Na}$ Fig. 10, vemos a curva de pressão contra volume de ar ocupado dentro do balão tipo globo que utilizamos em nossa prática. $\mathrm{O}$ ar foi injetado dentro do balão a partir de seringas graduadas em $\mathrm{mL}$ enquanto a pressão foi monitorada pelos dois sensores (Freescale e Vernier) ao mesmo tempo. O balão inflou até seu rompimento. Como o volume de ar é injetado a partir de seringas na pressão ambiente, é sabido que o ar dentro do balão sofrerá uma redução de volume prevista pela Lei de Boyle. Esta correção já está contabilizada no gráfico mostrado. Vemos pelo gráfico da Fig. 10 que a pressão dentro do balão é sempre maior que a atmosférica e passa por um valor mínimo para depois voltar a subir em função do aumento de volume, conforme encontrado também por (LANG; LEVIN, 2004) em outro balão similar. O trabalho de (LANG; LEVIN, 2004) informa que esse resultado é contra intuitivo, pois as pessoas costumam pensar que quanto maior for o volume, tanto maior deveria ser a pressão no interior do balão. Isto não ocorre para boa parcela do gráfico obtido.

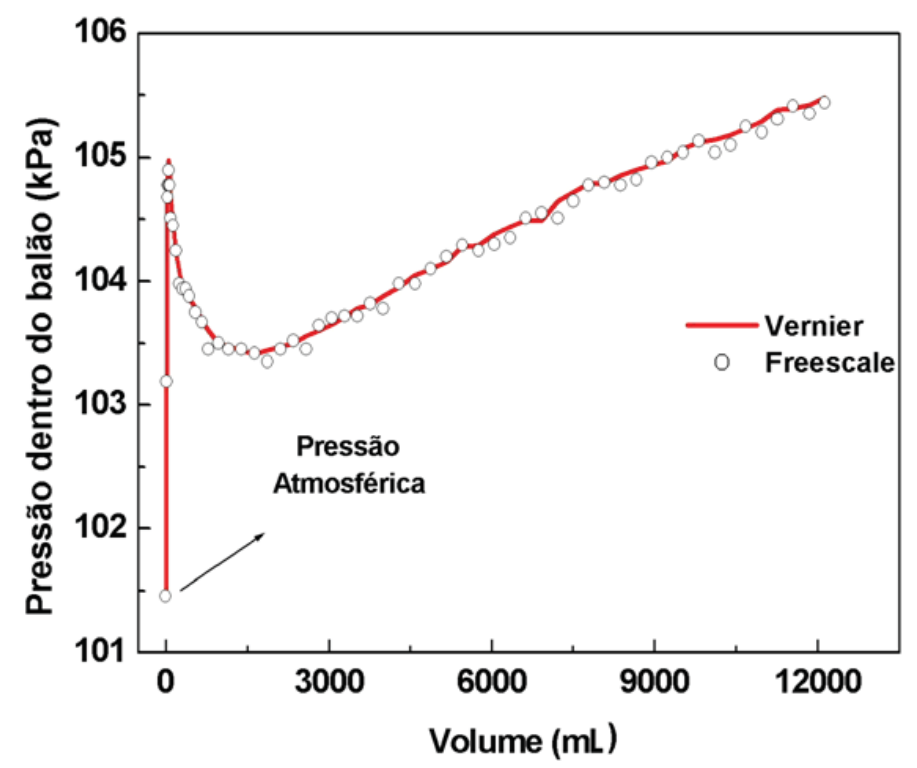

Fig. 10 - Variação de pressão no interior do balão em função do volume de ar injetado no seu interior. Comparação entre o desempenho do sensor apresentado neste trabalho com o sensor comercial da marca Vernier. 
A boa concordância entre os dados experimentais medidos pelos dois sensores é atestada pelo desvio quadrático médio de $0,0653 \mathrm{kPa}$ e desvio percentual máximo de $0,18 \%$ para toda a banda de volume testada. Estes valores de desvios são, na verdade, menores que a resolução do sensor, que no caso de conversão analógico digital de 10 bits fica no valor de $0,26 \mathrm{kPa}$.

Pelo gráfico da Fig. 10, vemos que um balão de látex de menor volume pode ter pressão interna maior do que outro balão que comporta uma quantidade de ar maior. Na possibilidade de haver a transferência espontânea de ar de um balão para o outro através da abertura de uma válvula de intercomunicação, (LANG; LEVIN, 2004) mostram a concretização de uma proposição contraintuitiva na qual o balão menor é que supre o maior com uma porção de ar até se estabelecer o equilíbrio de pressão. Propomos uma medida dinâmica deste evento. Utilizamos o balão globo $(1500 \mathrm{~mL})$ com volume de ar cinco vezes maior que o balão espaguete (300 mL). A Fig. 11 aponta a dinâmica da variação de pressão no sistema antes e após a abertura da válvula de duas vias que faz a intercomunicação entre os balões.

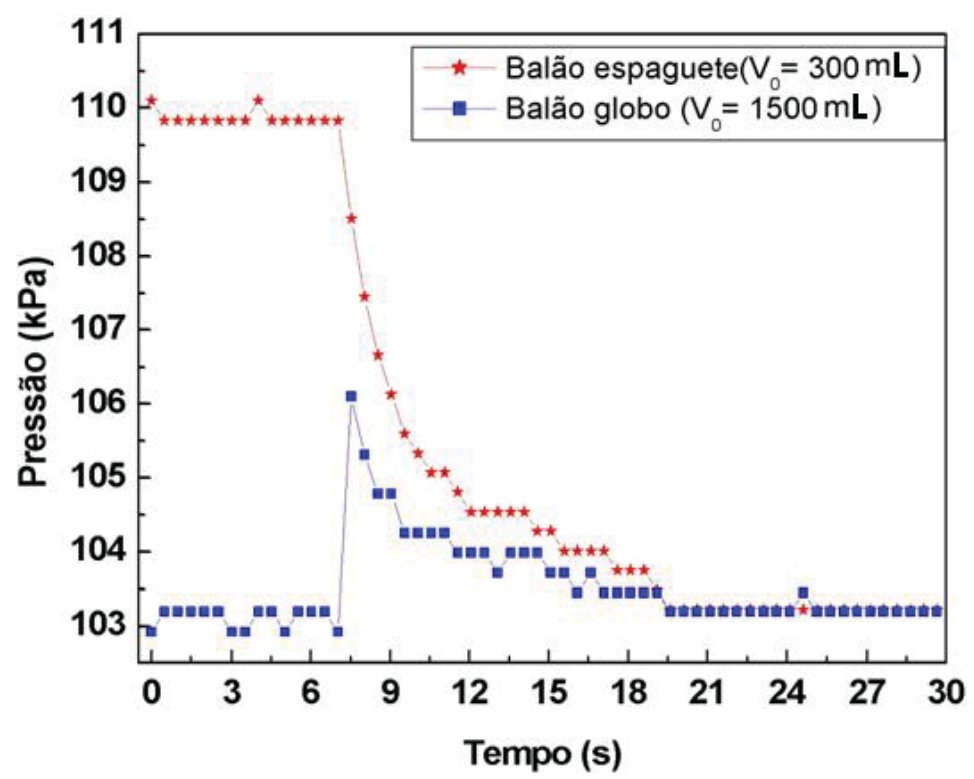

Fig. 11 - Variação de pressão no interior dos balões em função do tempo após a abertura da válvula de duas vias.

É possível ver que, logo da abertura da válvula, a isonomia de pressão no circuito fechado não se dá imediatamente mostrando que o sistema possui certa 
impedância. Pode-se notar um crescimento repentino da pressão sentida pelo sensor que monitora o balão globo e depois podemos ver o patamar de pressão final atingido. Ao final do processo, se estabelece o equilíbrio de pressão, contudo, os dois balões não apresentam o mesmo volume final, indicando a existência de deformações plásticas nas paredes de látex durante o processo conforme discutido por (LANG; LEVIN, 2004; LEVIN; LANG, 2004).

\section{Considerações finais}

Apresentamos as características de constituição física e funcionamento de um sensor de pressão manométrico útil para ensino de física experimental. Com a grande oferta de sensores de pressão no mercado atual e seu relativo baixo custo financeiro, se pode pensar num maior espaço para este dispositivo dentro de um laboratório didático de física experimental.

A prática ilustrativa mostrada pode ser desenvolvida em uma sala de aula comum com recursos de baixo custo. As características do sensor de pressão podem ser aproveitadas tanto com a finalidade de inovar e incrementar práticas de laboratório como quanto para incentivar alunos a trabalhar em projetos interdisciplinares. Ao trabalhar em projetos similares, o estudante pode desenvolver conhecimentos sobre sensores eletrônicos, microcontroladores (e sua programação computacional) e os limites de validade da informação numérica experimental.

\section{Referências}

BORGES, T. A. Novos rumos para o laboratório escolar de ciências. Caderno Brasileiro de Ensino de Física, v. 19, n. 3, p. 291-313, 2002.

CAVAlCANTE, M. A.; TAVOLARO, C. R. C.; MOLISANI, E. Física com Arduíno para iniciantes. Revista Brasileira de Ensino de Física, v. 33, n. 4, p. 4503, 2011.

GUADAGNINI, P. H.; BARLETTE, V. E. Um termômetro eletrônico de leitura direta com termistor. Revista. Brasileira de Ensino Física, n. 27, p. 369-375, 2005.

GUADAGNINI, P. H.; ROCHA, F. S. D.; BARLETTE, V. E. Projeto de um sensor eletrônico baseado em extensometria para medição de força. Latin-American Journal of Physics Education, v. 5, n. 4, p. 753-762, 2011. 
HAAG, R.; ARAUJO, I. S.; VEIT, E. A. Por que e como introduzir aquisição automática de dados no laboratório didático de Física? Física na Escola, v. 6, n. 1, p. 69-74, 2005.

KIRIANAKI, N. et al. Data Acquisition and Signal Processing for Smart Sensors. Baffins Lane: John Wiley \& Sons Ltd, 2002.

LANG, F. S.; LEVIN, Y. Pressão e volume em balões de festa: podemos confiar em nossa intuição? Caderno Brasileiro de Ensino de Física, v. edição especial, p. 143-153, dez. 2004.

LEVIN, Y.; LANG, F. S. Two rubber balloons: Phase diagram of air transfer. Physical Review E, v. 69, p. 051108, 2004.

METCALF, S. J.; TINKER, R. F. Probeware and handhelds in elementar and middle school science. Journal of Science Education and Technology, v. 13, n. 1, p. 43-49, 2004.

PÉREZ, D. G. et al. Tiene sentido seguir distinguiendo entre aprendizaje de conceptos, resolución de problemas de lápiz y papel y realización de prácticas de laboratorio? Enseñanza de las Ciencias, v. 17, n. 2, p. 311-320, 1999.

ROCHA, F. S. D.; GUADAGNINI, P. H. Sensor sonar de movimento para o ensino de física experimental. Latin-American Journal of Physics Education, v. 4, n. 24, p. $306-315,2009$.

SOKOLOFF, D. R. Active learning of introductory optics: Strategies for the U.S. and the developing World. Latin-American Journal of Physics Education, v. 6, n. Sup. I, 2012.

SOKOLOFF, D. R.; BENEGAS, J. Manual de Entrenamiento. 2do. Taller Regional del Cono Sur sobre Aprendizaje Activo de la Física: Mecánica. Córdoba, Argentina, 2009.

SOKOLOFF, D. R.; LAWS, P. W.; THORNTON, R. K. RealTime Physics: active learning labs transforming the introductory laboratory. European Journal of Physics, v. 28, p. 83-94, 2007.

SOUZA, A. R. et al. A placa Arduíno: uma opção de baixo custo para experiências de física assistidas pelo PC. Revista Brasileira de Ensino de Física, v. 33, n. 1, p. 1702, 2011. 
SZE, S. M. Semiconductor Sensors. New York: John Wiley \& Sons, 1994.

\section{Anexo}

Apresentamos o código de programação computacional que foi desenvolvido neste trabalho visando à leitura dos níveis de tensão elétrica variáveis $\mathrm{CC}$ do sensor de pressão através da placa Arduíno, o processamento desta informação e sua transmissão via porta serial do computador. É importante atentar que o sinal CC variável que é colhido do sensor dinamicamente é convertido em sinal digital pelo conversor de 10 bits presente nas portas A3 e A4 do microcontrolador. Após esta conversão, a programação realiza uma operação matemática que converte o número binário em unidades de $\mathrm{kPa}$ antes de seguir para o computador via porta serial.

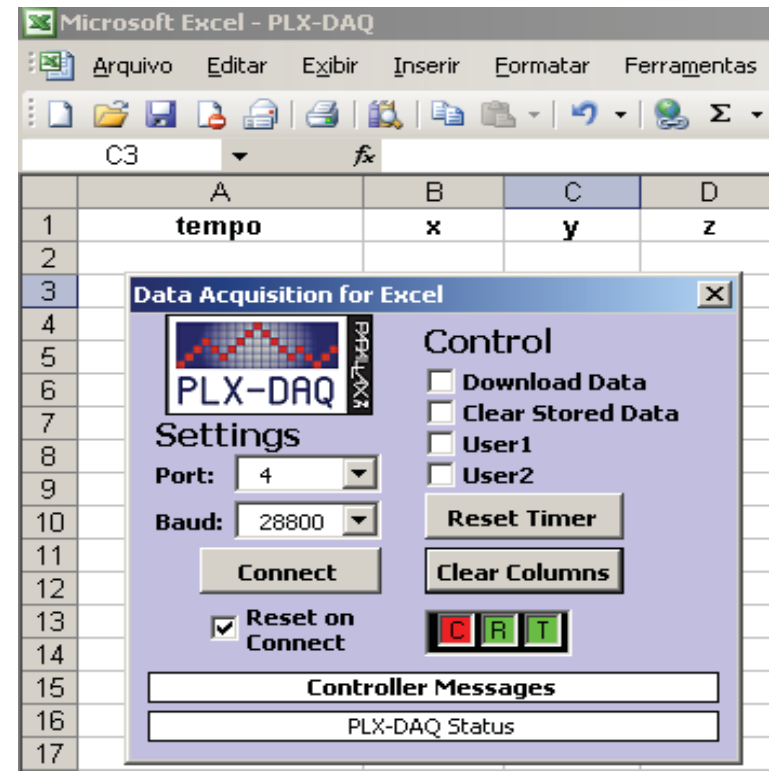

Fig. 12 - Caixa de controle inicial do programa PLX-DAQ. Importante definir a taxa "Baund" e a porta serial por onde será transmitida a informação.

O programa computacional $P L X-D A Q$ desenvolvido e disponibilizado gratuitamente pelo site da empresa PARALLAX INC. constitui uma ferramenta útil para viabilizar a leitura dos dados enviados pela placa Arduíno via porta serial. O 
programa $P L X-D A Q$ pode ler até 26 canais de dados simultaneamente, em tempo real, através de uma taxa de até $128 \mathrm{kbps}$. Os números que chegam do Arduíno são dispostos numa tabela do programa Excel, disponibilizando assim o gráfico atualizável da grandeza física que se esteja medindo (em nosso caso pressão). Além da programação revelada neste anexo, que viabiliza a comunicação do Arduíno com o PLX-DAQ, o site da empresa PARALLAX INC (https://classic.parallax.com/tabid/393/Default.aspx) disponibiliza outros exemplos que ilustram a potencialidade desta ferramenta. Na Fig. 12 mostramos a caixa de controle principal do $P L X-D A Q$.

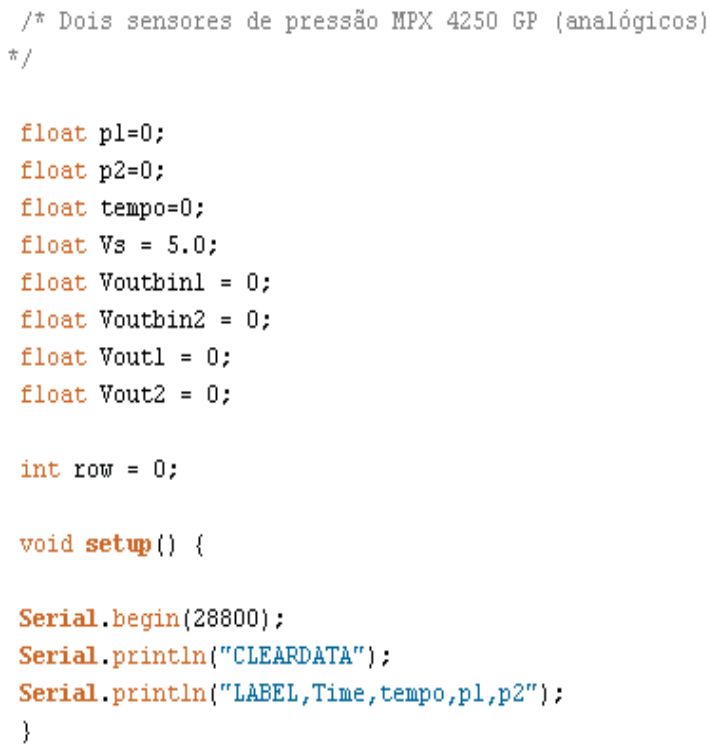

Fig. 13 - Primeira etapa do código computacional utilizado no microcontrolador Atmega 328 embarcado na placa Arduíno.

No código apresentado na Fig. 13 vemos primeiro a definição das variáveis importantes p1, p2, tempo, VS, Voutbin1, Voutbin2, Vout1 e Vout2. Em seguida temos a definição da taxa de transferência de dados "Serial.begin (28800)" que deve ser a mesma a ser escolhida na caixa de controle do $P L X-D A Q$ (veja a Fig. 12). Este valor pode ser aumentado até 128000 significando aumento da capacidade de dados transferidos por segundo. No "void setup", encontramos o início da formatação dos dados que são enviados pela serial e devidamente 
interpretados pelo $P L X$-DAQ. Esta formatação é específica e não pode ser alterada em sua estrutura para que os dados enviados sejam corretamente interpretados pelo $P L X-D A Q$.

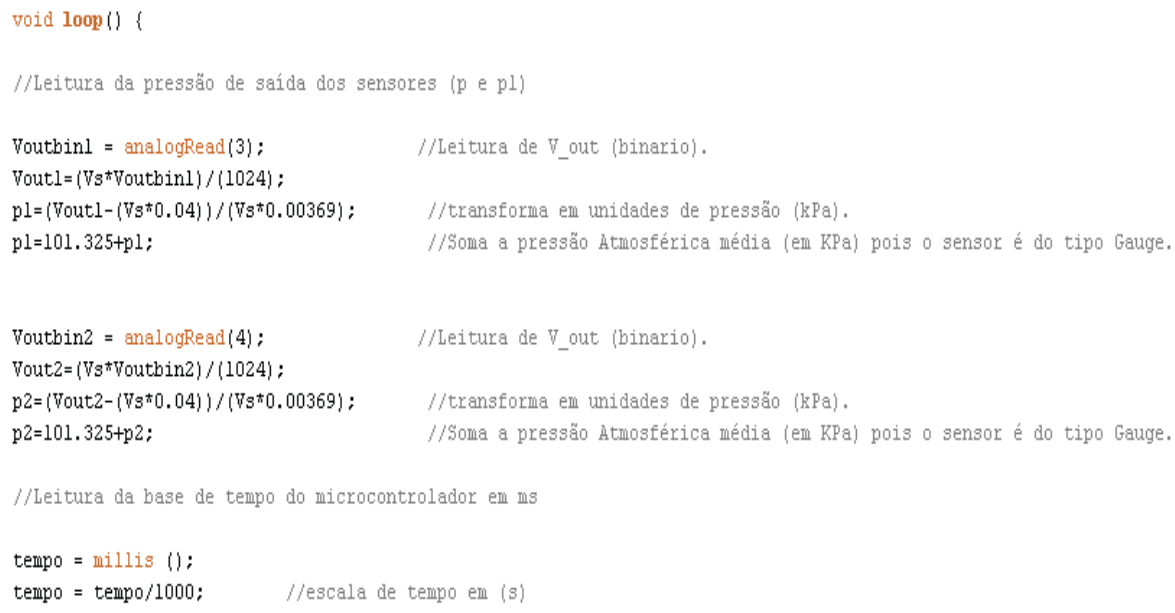

Fig. 14 - Segunda etapa do código computacional utilizado no microcontrolador Atmega 328 embarcado na placa Arduíno.

No comando serial.println, devemos indicar todas as variáveis que devem ser enviadas para a tabela do Excel, em nosso caso, Time, tempo, p1 (pressão sentida pelo sensor 1) e p2 (pressão sentida pelo sensor 2). Na Fig. 14, dentro do comando "void loop" vemos o comando analogRead que permite a leitura das informações vindas dos sensores de pressão pelas portas A3 (sensor 1) e A4 (sensor 2) e a conversão analógico-digital. É possível ver o conjunto de instruções matemáticas que traduzem a função transferência apontada na Fig. 6. A base de tempo do microcontrolador é lida na função millis que fornece o tempo em milissegundos. Os dados lidos e processados seguem para o Excel pelo comando serial.println. No exemplo que apresentamos, o "loop" se dá incrementando um contador de unidade em unidade até o limite de, por exemplo, 10000 (veja a Fig. 15). Esse valor representará o número de linhas que serão populadas com os dados medidos a cada $500 \mathrm{~ms}$. Uma vez alcançado este limite de 10000, a planilha volta para a primeira linha apagando os dados remanescentes e atualizando a informação. Segue assim indefinidamente. 
Dentro do ambiente de programação do código Arduíno o leitor deverá juntar todas as três etapas mostradas aqui nas Fig. 13, Fig. 14 e Fig. 15 em um só código sem interrupções e na ordem em que foi mostrado.

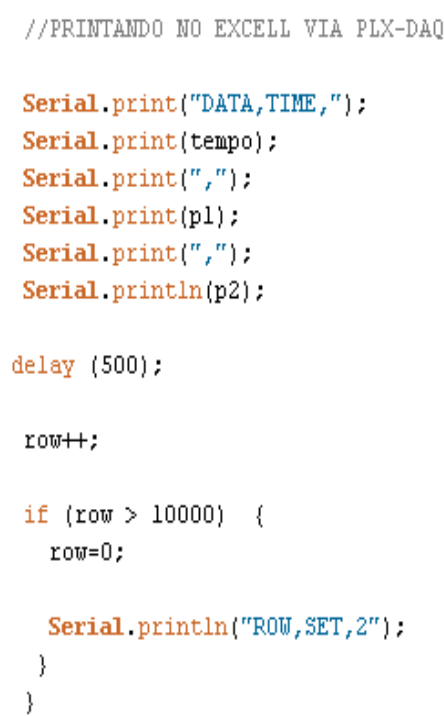

Fig. 15 - Terceira etapa do código computacional utilizado no microcontrolador Atmega 328 embarcado na placa Arduíno. 